\title{
AVALIAÇÃO DA QUALIDADE DE VIDA E VULNERABILIDADE AO ESTRESSE NO CONTEXTO HOSPITALAR
}

Fernanda Ottati

Mestre e doutoranda em Psicologia pelo Programa de Pós-Graduação Stricto Sensu em Psicologia da Universidade São Francisco. Docente da graduação e pós-graduação lato Sensu em Psicologia pela mesma instituição.

\author{
Vanessa Freitas \\ Psicóloga pela Universidade São Francisco.
}

\begin{abstract}
Resumo
O conceito de Qualidade de Vida é usado para medir as condições de vida de um ser humano, já a palavra vulnerabilidade refere-se à chance das pessoas estarem expostas ao adoecer. Objetivou-se analisar possíveis relações entre percepção de qualidade de vida e a vulnerabilidade ao estresse no trabalho em enfermeiros, técnicos e auxiliares de enfermagem que atuam na UTI e no setor de emergência de um hospital público. Utilizou-se o instrumento de qualidade de vida da Organização Mundial da Saúde, WHOQOL-Bref e a Escala de Vulnerabilidade ao Estresse no Trabalho. Encontrou-se, em relação ao setor de trabalho, diferença significativa apenas para Qualidade de vida total. Portanto pode-se afirmar que os profissionais que atuam na UTI possuem maior percepção de qualidade de vida quando comparados aos do SUS. Na correlação entre os dois instrumentos, o domínio meio ambiente do WHOQOL-Bref correlacionou-se negativa e significativamente com todos os fatores da EVENT, indicando que a percepção da pessoa em relação aos diversos aspectos do meio em que vive tem relação com a vulnerabilidade ao estresse.
\end{abstract}

Palavras-chave: enfermagem; hospital; qualidade de vida; estresse.

\section{EVALUATION OF QUALITY OF LIFE AND VULNERABILITY TO STRESS IN HOSPITAL CONTEXT}

\begin{abstract}
The concept of Quality of Life is used to measure the conditions of life of a human being, as the word vulnerability refers to the chance of being exposed to sick people. This study aimed to examine possible relationships between perceived quality of life and vulnerability to job stress in nurses, technicians and nursing assistants who work in the ICU and emergency department of a public hospital. We used the instrument of quality of life of the World Health Organization, WHOQOL-Bref and Scale Vulnerability to Stress at Work. Met in relation to the labor sector, a significant difference only for Quality of Life total. Therefore it can be said that the professionals who work in the ICU have a higher perception of quality of life when compared to the SUS. In the correlation between the two instruments, the environment domain of the WHOQOLBref correlated negatively and significantly with all factors EVENT, indicating that the perception of the person in relation to various aspects of the environment they live in is related to vulnerability to stress.
\end{abstract}

Keywords: nursing; hospital; quality of life; stress. 


\title{
EVALUACIÓN DE LA CALIDAD DE VIDA Y LA VULNERABILIDAD AL ESTRÉS EN EL CONTEXTO DEL HOSPITAL
}

\begin{abstract}
Resumen
El concepto de calidad de vida se utiliza para medir las condiciones de vida de un ser humano, como la vulnerabilidad palabra se refiere a la posibilidad de estar expuesto a enfermidades. Este estudio tuvo como objetivo examinar las posibles relaciones entre la calidad de vida percibida y la vulnerabilidad al estrés laboral en enfermeras, técnicos y auxiliares de enfermería que trabajan en el departamento de la UTI y de urgencias de un hospital público. Se utilizó el instrumento de calidad de vida de la Organización Mundial de la Salud, WHOQOL Bref y la Escala de Vulnerabilidade ao estresse no trabalho. Satisfecho en relación con el sector laboral, una diferencia significativa sólo para la calidad de vida total. Por lo tanto, se puede decir que los profesionales que trabajan en la UTI tienen una mayor percepción de la calidad de vida en comparación con el SUS. En la correlación entre los dos instrumentos, el dominio del medio ambiente de la WHOQOL-Bref correlación negativa y significativamente con todos los factores de evento, lo que indica que la percepción de la persona en relación con diversos aspectos del entorno en el que vivimos está relacionada con la vulnerabilidad a estrés.
\end{abstract}

Palabras clave: enfermería; hospital; calidad de vida; el estrés.

\section{INTRODUÇÃO}

O conceito de Qualidade de Vida é bastante amplo, usado para medir as condições de vida de um ser humano. Envolve e incorpora de forma complexa, a saúde física, mental, psicológica e emocional, além de relacionamentos sociais, nível de independência, crenças pessoais e a relação com aspectos significativos do meio ambiente. Embora não haja consenso ou uma definição amplamente adotada de qualidade de vida, alguns pesquisadores a definem como sensação íntima de conforto, bem-estar ou felicidade no desempenho de algumas funções (Fleck, 2008).

A Organização Mundial de Saúde (OMS), por exemplo, definiu qualidade de vida como sendo a compreensão da pessoa de sua postura na vida, no âmbito cultural e no processo de valores nos quais ela vive e em relação aos seus propósitos, possibilidades, status e aflições (Fleck, 2000). Para Luz, Tosta e Milaneze (2009) atualmente a qualidade de vida no trabalho pode ser compreendida como uma ideia envolvendo ocupação, empresas e pessoas, onde se destacam a preocupação com a satisfação do trabalhador, a eficácia organizacional e a participação dos trabalhadores nas decisões e problemas do trabalho. A satisfação no trabalho não pode estar separada da vida do indivíduo como um todo. Mais especificamente, o ambiente de trabalho dentro de uma Instituição Hospitalar caracteriza-se por um convívio diário com o sofrimento humano, o que demanda uma energia adicional nas tarefas a serem realizadas. 
Sendo assim, as pessoas que trabalham nestas instituições devem ser saudáveis tanto do ponto de vista físico, quanto do ponto de vista psicológico. As organizações que abrangem a preocupação com os funcionários se diferenciam de outras empresas em relação à produtividade e competitividade e se fundamentam principalmente na qualidade da atenção dada à pessoa e na satisfação da mesma com o serviço prestado

A preocupação com o sofrimento e a satisfação no trabalho de equipes de enfermagem teve início com argumentações sobre o modo como esses profissionais conseguiam suportar um trabalho tão desgastante, principalmente pela necessidade de conviverem com o sofrimento, dor e a morte de modo tão frequente (Guerrer \& Bianchi, 2011). Desta forma, o estresse tem sido um relevante aspecto também estudado nesse contexto.

O termo estresse ao longo do século XVII, era tido como aflição e adversidade, logo se tornou um fenômeno usado para caracterizar a soma de angústia, tensão e desconforto, que predomina na realidade e sociedade atual. No século seguinte, estresse passou a ser visto como a ação de força, pressão ou influência, muito além da capacidade de uma pessoa conseguir suportar, fazendo com que ela se curve perante a realidade (Lipp, 1996). Perkins (1982 citado por Miguel \& Noronha, 2007), assim como Lipp (2010a), enfatizam que o estresse não aparece de repente, e sim progressivamente onde os acontecimentos do dia a dia podem gerar algum incômodo, mesmo que este seja brando, de modo que, não são apenas os eventos devastadores que ocasionam desordem e estresse, mas os fatos do cotidiano também podem acarretar algum estresse e risco.

De acordo com Harbs, Rodrigues e Quadros (2008), por lidar constantemente com o sofrimento, a equipe de enfermagem apresenta alto risco de estresse, pois vive uma realidade de trabalho desgastante causada pela intensidade de exposição a cargas físicas e psíquicas. O contexto hospitalar contribui para o surgimento do estresse que muitas vezes o indivíduo demora em perceber seu adoecimento, tornando a qualidade do atendimento ao paciente insatisfatório. Segundo Corrêa (2008), os profissionais de saúde e em especial, aqueles que se dedicam a trabalhar em unidades de emergência do Sistema Único de Saúde (SUS) e em Unidades de Tratamento Intensivo (UTI's) estão submetidos a um estresse fora de seu controle, acarretando inúmeros prejuízos que, por sua vez, acabam por ser repassados aos pacientes à medida que sua 
concentração, capacidade de decisão, raciocínio, reflexos, serenidade e sensibilidade, encontram-se bastante comprometidos.

Um ponto importante no surgimento do estresse diz respeito à vulnerabilidade do indivíduo em apresentar ou não estresse excessivo. Vulnerabilidade se refere a uma predisposição a reagir de modo intenso, por meio de reações psicológicas e físicas, a situações estressantes (Pereira \& Tricoli, 2010). De acordo com Priel e Pessini (2009), todos os seres humanos podem se encontrar em alguma situação de vulnerabilidade ou que seja desfavorável em relação à outra pessoa ou pelo ambiente em que está inserido, visto que a vulnerabilidade se refere a uma tendência a reagir de modo intenso, por meio de reações psicológicas e físicas, a situações estressantes.

Existem dois componentes principais da vulnerabilidade, 0 inato e 0 adquirido. A vulnerabilidade inata é aquela que representa o ambiente interno e a neurofisiologia do organismo, ou seja, está nos genes. O componente adquirido é influenciado pelo ambiente externo, como doenças físicas, experiências familiares, relacionamentos na adolescência e outros. Quando as eventualidades da vida se tornam suficientes para desencadear um episódio de estresse, a pessoa é considerada muito vulnerável, enquanto que as pessoas com menor grau de vulnerabilidade precisam de acontecimentos mais intensos para induzir um episódio de doença (Lipp, 2010b).

Assim, indivíduos mais vulneráveis tendem a ser mais sensíveis ao estresse e sentem a sobrecarga mesmo em situações consideradas normais pela maioria das pessoas. O que ocorre nesse caso, é que sua capacidade para solucionar alguns problemas não é suficiente para reestabelecer a homeostase, seu equilíbrio fisiológico, seja porque o trauma é muito grande, difícil de solucionar, seja porque sua disposição e capacidade de enfrentamento não estão adequadas àquela situação. Então, visto que nesse caso normalmente a pessoa não encontra solução para o problema, o sofrimento se prolonga e a pessoa não vê saída para a situação em que se encontra (Macedo, 2010).

Em seu estudo, Salles (2005) objetivou avaliar a percepção de qualidade de vida de 125 auxiliares e técnicos de enfermagem em UTI's, sendo a maioria do sexo feminino, com mais de dois empregos e que trabalham há mais de cinco anos em UTI's. O instrumento utilizado na pesquisa foi o questionário WHOQOL100, composto de seis Domínios: Físico, Psicológico, de Independência, Relações Sociais, Ambiente, Espiritualidade/Religião/Crenças Pessoais. Os resultados 
mostraram a insatisfação com a realidade local em relação às condições de segurança física, com os meios de transporte e enfatizaram a urgente necessidade de melhoria salarial. O resultado positivo das médias dos domínios parece ter sido impulsionado pelo bom relacionamento interpessoal do grupo, sua capacidade para o trabalho, a autoestima e a fé como elemento essencial na sua vida.

Corrêa (2008), em sua pesquisa, buscou evidências de validade baseada nas relações com outras variáveis para a Escala de Vulnerabilidade ao Estresse no Trabalho (EVENT) por meio da correlação entre os fatores da EVENT e o Instrumento Abreviado de Qualidade de Vida da Organização Mundial da Saúde (WHOQOL-Bref). A amostra continha 117 profissionais da área de enfermagem, auxiliar de enfermagem, técnico de enfermagem e enfermeiros que trabalhavam em hospitais privados, privados e com convênio do Sistema Único de Saúde (SUS) e em serviço público na área de saúde pública de um município do Estado de São Paulo. A faixa etária dos participantes variou de 20 a 56 anos. Foi encontrada correlação negativa em todas as dimensões indicando que as escalas utilizadas mostram resultados inversos caracterizando que quanto maior for a vulnerabilidade ao estresse no trabalho menor é a qualidade de vida do trabalhador. Também foi encontrado que nem todas as dimensões dos dois instrumentos correlacionaram-se significativamente.

O estudo de Oswaldo (2009), buscou novas evidências de validade baseada na relação com outras variáveis da Escala de Vulnerabilidade ao Estresse no Trabalho (EVENT) com o Inventário de Stress para Adultos de Lipp (ISSL), Escala Coping Ocupacional (ECO), Escala de Depressão (EDEP) e Instrumento Abreviado de Qualidade de Vida (WHOQOL-Bref) em gestores nas áreas da saúde, educação e empresarial. Foram participantes 185 gestores, sendo da área empresarial $38,9 \%$, educação $33,0 \%$ e saúde $28,1 \%$, trabalhadores de instituições educacionais (escolas municipais e uma Universidade privada), dois hospitais (um público e outro privado) e duas empresas do ramo metalúrgico e cinco prestadoras de serviços, todas localizadas no interior do Estado de São Paulo. A idade dos participantes variou de 21 a 69, sendo a maioria feminino $(60,0 \%)$ e casado $(65,0 \%)$. Foi encontrada correlação negativa e de magnitude baixa e significativa entre os fatores da EVENT (Clima e Funcionamento, Pressão no Trabalho e Infraestrutura e Rotina) e todas as dimensões do WHOQOL-Bref (Físico, Psicológico, Relações Sociais, Meio Ambiente e Qualidade de Vida Geral). 
Nas dimensões da WHOQOL-Bref e as fases de estresse, os sujeitos que se encontram nas fases de Resistência e Quase-Exaustão, apresentaram limitações em todas as dimensões da WHOQOL-Bref, comprometimento com a qualidade de vida em relação aos sujeitos sem estresse.

Furlan (2011) buscou investigar a vulnerabilidade ao estresse laboral e a qualidade de vida em trabalhadores, além de também buscar a relação entre os construtos e verificar as diferenças de média em relação à idade, sexo, turno, estado civil, escolaridade e tempo de empresa. A amostra foi composta por 109 operadores de produção de uma empresa multinacional Francesa do ramo automobilístico, localizada no interior de São Paulo. Foi verificado que, embora os homens tenham apresentado maiores médias nos fatores da EVENT e em três domínios da WHOQOL-Bref, essas diferenças não foram significativas, podendo este ter sido afetado pela maior quantidade de homens. Em relação ao domínio psicológico da WHOQOL-Bref, os resultados obtidos apontaram que, quanto menor é a idade, maior é a qualidade de vida no fator psicológico. Os resultados no fator clima da EVENT apontaram que, quanto maior o tempo de empresa, maior a vulnerabilidade. Em relação às médias do estado civil, foi possível verificar que na amostra referente aos não casados a vulnerabilidade ao estresse era maior que na amostra de casados nos fatores clima e pressão no trabalho. Para os resultados da WHOQOL-Bref, foi possível verificar que a qualidade de vida estava maior na amostra de não casados nos domínios meio ambiente, relações sociais e físico.

Tendo em vista o exposto, o presente estudo teve como objetivo analisar possíveis relações entre percepção de qualidade de vida e a vulnerabilidade ao estresse no trabalho em enfermeiros, técnicos e auxiliares de enfermagem que atuavam na UTI e no setor de emergência (SUS) de um hospital público, bem como identificar eventuais diferenças relacionadas ao setor de trabalho no que se refere à percepção de qualidade de vida e a vulnerabilidade ao estresse no trabalho.

\section{MÉTODO}

\section{Participantes}

Participaram da pesquisa 41 funcionários de um hospital público localizado no interior de São Paulo. Destes, $85,4 \%(N=35)$ eram do sexo feminino e $14,6 \%$ $(\mathrm{N}=6)$ do sexo masculino. A idade dos participantes variou entre 20 e 52 anos 
sendo a média 32,78 ( $\mathrm{DP}=8,02)$. Em relação aos setores de atuação, os participantes distribuíram-se da seguinte forma: 53,7\% ( $N=22)$ atuavam no setor de emergência (SUS) e 46,3\% ( $N=19)$ na UTI.

\section{Instrumentos}

Instrumento de Qualidade de Vida da Organização Mundial da Saúde (WHOQOLBref)

De acordo com Fleck (1998), o WHOQOL-Bref foi desenvolvido pela Organização Mundial de Saúde (OMS), devido à necessidade de um instrumento mais curto, que demandasse pouco tempo para o preenchimento e que preservasse características psicométricas satisfatórias. O instrumento original WHOQOL-100, é composto de cem questões referente a seis domínios: físico, psicológico, nível de independência, relações sociais, meio ambiente e espiritualidade/religiosidade/crenças pessoais (Oliveira \& França, 2005). Já o WHOQOL-Bref por ser uma versão abreviada, é constituído por vinte e seis questões objetivas formuladas para uma escala de respostas do tipo Likert, com uma escala de intensidade (nada - extremamente), capacidade (nada completamente), frequência (nunca - sempre) e avaliação (muito insatisfeito muito satisfeito / muito ruim - muito bom). O instrumento é constituído por quatro domínios da qualidade de vida, quais sejam: capacidade física, o bemestar psicológico, as relações sociais e o meio ambiente onde o indivíduo está inserido (Fleck, 2008). A escala é positiva, isto é, quanto mais alto o escore, melhor a qualidade de vida naquele domínio.

\section{Escala de Vulnerabilidade ao Estresse no Trabalho (EVENT)}

A EVENT foi desenvolvida por Sisto, Baptista, Noronha e Santos (2007) e é constituída por 40 itens relacionados a situações geradoras do estresse, onde a pessoa deve assinalar os itens que causam incômodo a partir de uma escala Likert com opções de intensidade que são de "nunca" (1), "às vezes" (2), "frequentemente" (3). Seu objetivo é avaliar o quanto as situações do cotidiano do trabalho interferem no modo de agir do indivíduo, podendo caracterizar certa fragilidade.

Para a pontuação somam-se os itens assinalados, sendo a pontuação mínima de 40 e a máxima de 120 na escala total; mostrando que quanto maior a pontuação, maior é a vulnerabilidade ao estresse. Os itens estão divididos em 
três fatores, Fator 1 (Clima e Funcionamento Organizacional), Fator 2 (Pressão no Trabalho) e Fator 3 (Infraestrutura e Rotina).

\section{Procedimento}

Após a autorização da instituição hospitalar, o projeto foi avaliado e aprovado para execução pelo Comitê de Ética em Pesquisa. A coleta foi realizada coletivamente em hora oportuna aos sujeitos, na própria instituição de saúde, após a explicação dos objetivos da pesquisa e assinatura do Termo de Consentimento Livre e Esclarecido. O primeiro instrumento respondido foi EVENT, seguido pelo WHOQOL-Bref e o tempo médio de aplicação foi de 40 minutos.

Análise de dados

Para tabulação dos dados, seguiu-se a correção dos instrumentos tal como indicado pelos autores dos instrumentos. A análise estatística utilizada foi a paramétrica, tendo sido realizado primeiro as análises descritivas e posteriormente $\mathrm{o}$ teste $\mathrm{t}$ de Student para comparar duas variáveis. Para verificação dos índices de correlações, foi utilizada a prova de Spearman.

\section{RESULTADOS E DISCUSSÃO}

Os dados foram organizados em planilha eletrônica (SPSS 20) e apresentados conforme a ordem dos objetivos propostos. A primeira análise apresentada refere-se a estatística descritivas dos instrumentos, conforme Tabela 1.

Tabela 1.

Estatística descritiva da EVENT e WHOQOL-Bref para amostra total

\begin{tabular}{cccccc}
\hline & $\mathrm{N}$ & Mínimo & Máximo & Média & $\mathrm{DP}$ \\
\hline Clima e funcionamento & 41 & 21 & 44 & 30,34 & 5,96 \\
Pressão no trabalho & 41 & 17 & 35 & 27,32 & 4,88 \\
Infraestrutura e rotina & 41 & 12 & 27 & 16,58 & 3,35 \\
Event total & 41 & 51 & 105 & 74,24 & 12,02 \\
\hline Físico & 41 & 8,57 & 19,43 & 14,86 & 2,11 \\
Psicológico & 41 & 9,33 & 19,33 & 15,14 & 2,00 \\
Relações sociais & 41 & 9,33 & 18,67 & 15,19 & 2,25 \\
Meio ambiente & 41 & 8,50 & 16,00 & 12,82 & 2,08 \\
QV total & 41 & 8,00 & 20,00 & 14,44 & 3,15 \\
\hline
\end{tabular}

A análise da EVENT revela que no Fator 1 a média foi de 30,34 ( $D P=5,96)$ sendo que a pontuação máxima permitida neste fator é de 48 pontos. Para o 
Fator 2, a média foi de $27,32(\mathrm{DP}=4,88)$ sendo que a máxima permitida seria de 39 pontos. Por fim o Fator 3 apresentou média de 16,58 ( $D P=3,35)$, sendo o máximo possível de 33 pontos. Para a EVENT total os participantes apresentaram média de 74,24, sendo que o máximo permitido seria de 120 pontos. Cabe ressaltar que nesse instrumento, quanto maior a pontuação maior a vulnerabilidade ao estresse. Esses achados revelam que os participantes apresentam uma tendência mais próxima a vulnerabilidade, especificamente em dois fatores, Clima e funcionamento organizacional e Pressão no trabalho, nos quais as médias foram mais próximas ao máximo permitido de pontuação.

É importante ressaltar que, no WHOQOL-Bref, quanto maior a pontuação, melhor é a percepção da qualidade de vida, sendo portanto o contrário do que acontece na EVENT. Os dados permitem inferir que nesta amostra específica, a percepção de qualidade de vida em todos os domínios, apresenta-se na média. Segundo Corrêa (2008), a qualidade de vida se manifesta além da qualidade de saúde da pessoa, suas oportunidades e limitações individuais e coletivas, representando o processo de satisfação de suas necessidades com cuidados físicos e culturais.

Em relação ao setor de trabalho, foi realizada a diferença de média por meio do teste $t$ de Student, que revelou diferença significativa apenas para a Qualidade de vida total, sendo que os trabalhadores do SUS apresentaram média mais baixa que os da UTI, como indica a Tabela 2 .

No WHOQOL-Bref, em qualidade de vida total, a média dos participantes que atuam no SUS foi de $13,45(\mathrm{DP}=3,22)$ e a média dos que atuam na UTI foi de $15,58(D P=2,71)$, sendo encontrada a diferença estatisticamente significativa nessa dimensão. Portanto, pode-se afirmar que os profissionais que atuam na UTI possuem maior percepção de qualidade de vida quando comparados aos do SUS. Este dado diverge dos encontrados na literatura onde, de acordo com Salles (2005), a UTI por ser um ambiente permeado de incertezas torna-se um gerador de estresse aos profissionais da equipe multidisciplinar; complementando, Corrêa (2008) relata que os profissionais que atuam em Unidades de Tratamento Intensivo (UTI's) estão submetidos a um estresse fora de seu controle, acarretando inúmeros prejuízos que, por sua vez, acabam por ser repassados aos pacientes à medida que sua concentração, capacidade de decisão, raciocínio, reflexos, serenidade e sensibilidade, encontram-se bastante comprometidos. 
Tabela 2.

Diferença de média (t de Student) em relação ao setor de trabalho

\begin{tabular}{ccccccc}
\hline & Setor & $\mathrm{N}$ & Média & DP & $\mathrm{t}$ & $p$ \\
\hline Clima e funcionamento & SUS & 22 & 30,72 & 6,18 & 0,441 & 0,661 \\
& UTI & 19 & 29,89 & 5,82 & & \\
Pressão no trabalho & SUS & 22 & 26,59 & 5,27 & $-1,026$ & 0,311 \\
& UTI & 19 & 28,15 & 4,36 & & \\
Infraestrutura e rotina & SUS & 22 & 15,86 & 3,25 & $-1,506$ & 0,140 \\
& UTI & 19 & 17,42 & 3,35 & & \\
Event total & SUS & 22 & 73,18 & 12,17 & $-0,604$ & 0,549 \\
Físico & UTI & 19 & 75,47 & 12,05 & & \\
& SUS & 22 & 14,90 & 2,43 & 0,145 & 0,886 \\
Psicológico & UTI & 19 & 14,81 & 1,74 & & \\
& SUS & 22 & 15,06 & 1,95 & $-0,264$ & 0,793 \\
Relações sociais & UTI & 19 & 15,22 & 2,09 & & \\
& SUS & 22 & 14,90 & 2,24 & $-0,849$ & 0,401 \\
Meio ambiente & UTI & 19 & 15,50 & 2,27 & & \\
& SUS & 22 & 12,86 & 1,80 & 0,152 & 0,880 \\
QV total & UTI & 19 & 12,76 & 2,42 & & \\
& SUS & 22 & 13,45 & 3,22 & $-2,264$ & 0,029 \\
& UTI & 19 & 15,58 & 2,71 & & \\
\hline
\end{tabular}

Por fim, foi realizada a correlação de Spearman entre os fatores da EVENT e os domínios da WHOQOL-Bref, sendo encontradas correlações negativas e significativas, conforme mostra a Tabela 3.

Tabela 3.

Correlação de Spearman entre EVENT e WHOQOL-Bref

\begin{tabular}{cccccc}
\hline & Físico & Psicológico & $\begin{array}{c}\text { Relações } \\
\text { sociais }\end{array}$ & $\begin{array}{c}\text { Meio } \\
\text { ambiente }\end{array}$ & QV \\
$\begin{array}{c}\text { Clima e } \\
\text { funcionamento } \\
\begin{array}{c}\text { Pressão no } \\
\text { trabalho }\end{array}\end{array}$ & $-0,125$ & $-0,295$ & $-0,378^{*}$ & $-0,593^{* *}$ & $-0,277$ \\
$\begin{array}{c}\text { Infraestrutura e } \\
\text { rotina }\end{array}$ & $-0,298$ & $-0,292$ & $-0,149$ & $-0,543^{* *}$ & 0,045 \\
$\begin{array}{c}\text { EVENT } \\
\text { total }\end{array}$ & $-0,288$ & $-0,450^{*}$ & $-0,321$ & $-0,650^{* *}$ & $-0,137$ \\
\hline
\end{tabular}

* A correlação é significativa no nível 0,05

**A correlação é significativa no nível 0,01. 
As magnitudes das correlações variaram de baixas a altas (Sisto, 2007), sendo que o domínio psicológico correlacionou-se a EVENT Total, o domínio relações sociais com o Fator 1 (Clima e funcionamento organizacional) e, por fim, o domínio meio ambiente apresentou correlações com todos os fatores da EVENT.

Esses achados revelam que a vulnerabilidade ao estresse está relacionada ao meio ambiente de trabalho e também as relações sociais de forma que, quanto maior for a vulnerabilidade ao estresse para cada fator, menor será a percepção da qualidade de vida do indivíduo, pois as correlações foram negativas.

Os resultados evidenciam que, assim como nos estudos de Correa (2008), em pesquisa com profissionais da enfermagem, também encontrou correlações negativas entre a EVENT e WHOQOL-Bref. Igualmente, a pesquisa de Furlan (2011), por meio da utilização dos mesmos instrumentos, encontrou várias correlações negativas, constatando que quanto maior a vulnerabilidade ao estresse principalmente no que se refere à estrutura do ambiente de trabalho e a relação com seus superiores e colegas, os profissionais (enfermeiros, auxiliares e técnicos de enfermagem) tendem a apresentar menor qualidade de vida. $E$, a pesquisa de Oswaldo (2009), também encontrou correlações significativas e negativas entre os fatores da EVENT e dimensões do WHOQOL-Bref. Desta forma, pode-se considerar a fundamentação dos construtos avaliados, sendo esperadas as relações negativas, por se tratarem de condições opostas (Fleck, 2008; Sisto \& cols, 2007). De forma geral, na presente pesquisa, observa-se que quanto mais os sujeitos se sentem vulneráveis ao estresse em relação as condições ambientais, menor é a percepção da qualidade de vida.

Segundo Corrêa (2008), o estresse a que são submetidos os profissionais de saúde e, em particular, àqueles que se dedicam a trabalhar em unidades de emergência do Sistema Único de Saúde (SUS) e em Unidades de Tratamento Intensivo (UTI's) estão fora de seu controle; impõem-lhes inúmeros prejuízos que, por sua vez, acabam por ser repassados aos pacientes à medida que sua concentração, capacidade de decisão, limiar de irritabilidade, raciocínio, reflexos, serenidade, sensibilidade, encontram-se bastante comprometidos.

Pôde-se observar através dos dados obtidos na presente pesquisa, que os participantes que atuavam na UTI apresentaram maior percepção de qualidade de vida em relação aos que atuavam no SUS, demonstrando que embora 
estejam em um ambiente onde lidam com maior frequência com a morte, não vivenciam o mesmo estresse que a equipe do SUS ao trabalhar em constante pressão. Torna-se importante ressaltar que a vulnerabilidade ao estresse e a percepção de qualidade de vida estão interligadas, e este tema precisa ser mais explorado, pois ajudará os profissionais que atuam na área da saúde a refletir sobre atitudes que possam promover menos estresse e melhor qualidade de vida no contexto hospitalar.

\section{CONSIDERAÇÕES FINAIS}

Torna-se importante ressaltar que a vulnerabilidade ao estresse e a percepção de qualidade de vida estão interligadas, e este tema precisa ser mais explorado, pois ajudará os profissionais que atuam na área da saúde a refletir sobre atitudes que possam promover menos estresse e melhor qualidade de vida no contexto hospitalar. Dentre as contribuições encontradas neste estudo está o fato de que, no setor de trabalho, foi encontrada diferença significativa apenas para a Qualidade de vida total. Portanto, pode se afirmar que os profissionais que atuam na UTI possuem maior percepção de qualidade de vida quando comparados aos do SUS. Na correlação entre os dois instrumentos, o domínio meio ambiente correlacionou-se negativa e significativamente com todos os fatores da EVENT. Esses achados revelam que a vulnerabilidade ao estresse está relacionada ao meio ambiente, de forma que, quanto maior for a vulnerabilidade ao estresse para cada fator, menor será a percepção da qualidade de vida no domínio meio ambiente, o qual representa as relações pessoais, a atividade sexual, os recursos financeiros, a segurança física e proteção, os transportes, as oportunidades de lazer, a religião, dentre outros.

De acordo com a literatura, os profissionais que atuam na UTI são considerados mais vulneráveis ao estresse (Corrêa, 2008; Salles, 2005). Esta informação diverge do resultado da presente pesquisa que, ao comparar profissionais do SUS e da UTI, encontrou que os do SUS apresentaram maior vulnerabilidade ao estresse. Mas, é importante considerar que algumas variáveis não foram estudadas, como por exemplo, as condições físicas do ambiente de trabalho destes funcionários, bem como os relacionamentos entre chefia e colegas, que são importantes elementos para explicação dos fenômenos estudados. 
Com relação às limitações do presente estudo destaca-se: a pequena amostra, o fato de este ter sido realizado apenas em um hospital público e de não ter sido efetuado a comparação entre os hospitais públicos e particulares. Por meio dos resultados obtidos no presente estudo, pode-se verificar a necessidade de se trabalhar tais aspectos no ambiente hospitalar, com o intuito de melhorar a qualidade de vida da equipe de enfermagem e torná-la menos vulnerável a situações de estresse, independente da área em que atuem. Vale ressaltar a importância do papel do psicólogo no contexto hospitalar, trabalhando as questões voltadas para a promoção da qualidade de vida e diminuição de ocorrência de estresse entre os funcionários. Um trabalho de reflexão realizado por um psicólogo, que envolva toda a equipe de saúde, é uma das necessidades mais prementes para fazer com que o hospital perca seu caráter meramente curativo para transformar-se em uma instituição que trabalhe não apenas com a reabilitação orgânica, mas também com o restabelecimento da dignidade humana (Camon, 2001).

Sugere-se a realização de novos estudos com estes profissionais da saúde, com amostras maiores, para melhor identificar as necessidades das equipes de enfermagem e aumentar o conhecimento sobre o assunto, com o intuito de incentivar o desenvolvimento de políticas públicas de saúde, com atenção direcionada aos profissionais que atuam no SUS e na UTI, garantindo melhor condição de trabalho para que possam desempenhar suas funções da melhor maneira possível.

\section{REFERÊNCIAS}

Camon, V. A. A. (2001). Psicologia hospitalar: Teoria e prática. São Paulo: Pioneira Psicologia.

Corrêa, R. Z. A. (2008). Estresse laboral e qualidade de vida na equipe de enfermagem: Evidências de validade. Dissertação de Mestrado, Universidade São Francisco, Itatiba.

Fleck, M. P. A. (1998). Versão em português dos instrumentos de avaliação de qualidade de vida (WHOQOL). Disponível em: http://www.ufrgs.br/psiq/whoqol.html. Acessado em 06 de Setembro de 2011. 
Fleck, M. P. A. (2000). O instrumento de avaliação de qualidade de vida da Organização Mundial da Saúde (WHOQOL-100): Características e perspectivas. Ciência e Saúde Coletiva, 5(1).

Fleck, M. P. A. (2008). A avaliação de qualidade de vida: Guia para profissionais da saúde. Porto Alegre: Artmed.

Furlan, A. (2011). Vulnerabilidade ao estresse laboral e qualidade de vida em trabalhadores. Trabalho de Conclusão de Curso, Universidade São Francisco, Itatiba.

Guerrer, L. F. J., \& Bianchi, F. E. R. (2011). Estresse dos enfermeiros atuantes em UTI nas regiões do Brasil. Revista Eletrônica Trimestral de Enfermagem, 22.

Harbs, T. C.; Rodrigues, S. T. \& Quadros, V. A. S. (2008). Estresse da equipe de enfermagem em um centro de Urgência e emergência. Boletim de Enfermagem, 2(1), 41-56.

Lipp, M. E. N. (1996). Pesquisas sobre estresse no Brasil: Saúde, ocupações e grupos de risco. Campinas, SP: Papirus.

Lipp, M. E. N. (2010a). Mecanismos neuropsicofisiológicos do stress: Teoria e aplicações clínicas. $3^{\circ}$ ed. São Paulo: Casa do Psicólogo.

Lipp, M. E. N. (2010b). O Tratamento Psicológico do Stress. Em: Lipp, M. E. N. (2010). Mecanismos neuropsicofisiológicos do stress: Teoria e aplicações clínicas. $3^{\circ}$ ed. (pp. 187-192). São Paulo: Casa do Psicólogo.

Luz, M. M.; Tosta, T. B. C. K. \& Milaneze, C. (2009). Qualidade de Vida no Trabalho: O Caso dos Servidores do Ambulatório do Hospital Universitário da Universidade Federal de Santa Catarina. Disponível em: http://www.inpeau.ufsc.br/wp/v1/detalhe-artigos.php?id=1137. Acessado em 25 de Outubro de 2011.

Macedo, S. B. M. (2010). Estudo correlacional entre vulnerabilidade ao estresse no trabalho e traços de personalidade. Dissertação de Pós Graduação, Universidade São Francisco, Itatiba.

Miguel, F. K., \& Noronha, A. P. P. (2007). Estudo dos Parâmetros Psicométricos da Escala de Vulnerabilidade ao Estresse no Trabalho. Evaluar, 7, 1-18. 
Oliveira, P. M., \& França, A. C. L. (2005). Avaliação da gestão de programas de qualidade de vida no trabalho. Disponível em: http://www.scielo.br/pdf/raeel/v4n1/v4n1a05.pdf. Acessado dia 25 de Outubro de 2011.

Oswaldo, Y. C. (2009). Vulnerabilidade ao estresse no trabalho, Coping, depressão e qualidade de vida: Evidências de validade. Defesa apresentada ao Programa de Pós-Graduação, Universidade São Francisco, Itatiba.

Pereira, M. M. B., \& Tricoli, V. A. C. (2010). A influência do meio ambiente e de práticas parentais na vulnerabilidade ao stress. Em: Lipp, M. E. N. (2010). Mecanismos neuropsicofisiológicos do stress: Teoria e aplicações clínicas. $3^{\circ}$ ed. (pp. 67-70). São Paulo: Casa do Psicólogo.

Priel, M. R., \& Pessini, L. (2009). O conceito de vulnerabilidade no contexto das pesquisas envolvendo seres humanos. Revista Brasília Médica, 46(1), 5154.

Salles, E. P. (2005). Qualidade de vida do auxiliar e técnico de enfermagem em UTI's. Dissertação de Mestrado em Enfermagem. Universidade Federal de Goiás - Goiânia.

Sisto, F. F.; Baptista, M. N; Noronha, A. P. P. \& Santos, A. A. A. (2007). Escala de Vulnerabilidade ao Estresse no Trabalho (EVENT). São Paulo: Vetor.

Sisto, F. F. (2007). Delineamento correlacional. Em Baptista, M. N. \& Campos, D. C. (orgs.), Metodologias de pesquisa em ciências. (pp.90-101). Rio de Janeiro: LTC.

Contato: fernanda_itb@yahoo.com.br, vanessaadefreitas@gmail.com

Recebido em: 20/02/2013

Revisado em: 28/03/2013

Aceito em: 14/04/2013 